\title{
Weighted Radon transforms and first order differential systems on the plane
}

\section{R.G. Novikov}

CNRS (UMR 7641), Centre de Mathématiques Appliquées, Ecole Polytechnique, 91128 Palaiseau, France

e-mail: novikov@cmap.polytechnique.fr

\section{Abstract}

We consider weighted Radon transforms on the plane, where weights are given as finite Fourier series in angle variable. By means of additive Riemann-Hilbert problem techniques, we reduce inversion of these transforms to solving first order differential systems on $\mathbb{R}^{2}=\mathbb{C}$ with a decay condition at infinity. As a corollary, we obtain new injectivity and inversion results for weighted Radon transforms on the plane.

\section{Introduction}

We consider the weighted ray transforms $P_{W}$ defined by the formula

$$
P_{W} f(s, \theta)=\int_{\mathbb{R}} W\left(s \theta^{\perp}+t \theta, \theta\right) f\left(s \theta^{\perp}+t \theta\right) d t, \quad s \in \mathbb{R}, \theta=\left(\theta_{1}, \theta_{2}\right) \in \mathbb{S}^{1},
$$

where $\theta^{\perp}=\left(-\theta_{2}, \theta_{1}\right), W=W(x, \theta)$ is the weight, $f=f(x)$ is a test function, $x \in \mathbb{R}^{2}$, $\mathbb{S}^{1}$ is the unit circle in $\mathbb{R}^{2}$. Up to change of variables, $P_{W}$ is known also as the weighted Radon transform on the plane.

In this work one can always assume that

$$
W \in C\left(\mathbb{R}^{2} \times \mathbb{S}^{1}\right) \cap L^{\infty}\left(\mathbb{R}^{2} \times \mathbb{S}^{1}\right)
$$

Additional assumptions on $W$ will be formulated in the framework of context. In particular, in important particular cases $W$ is real-valued and strictly positive:

$$
W=\bar{W}, \quad W \geq c>0 .
$$

We recall that in definition (1.1) the product $\mathbb{R} \times \mathbb{S}^{1}$ is interpreted as the set of all oriented straight lines in $\mathbb{R}^{2}$. More precisely, if $\gamma=(s, \theta) \in \mathbb{R} \times \mathbb{S}^{1}$, then $\gamma=\left\{x \in \mathbb{R}^{2}\right.$ : $\left.x=s \theta^{\perp}+t \theta, t \in \mathbb{R}\right\}$ (modulo orientation) and $\theta$ gives the orientation of $\gamma$.

If $W \equiv 1$, then $P_{W}$ is known as classical ray (or Radon) transform on the plane. This transform arises, in partcular, in X-ray transmission tomography. If

$$
W(x, \theta)=\exp (-D a(x, \theta)), \quad D a(x, \theta)=\int_{0}^{+\infty} a(x+t \theta) d t
$$

where $a$ is a complex-valued sufficiently regular function on $\mathbb{R}^{2}$ with sufficient decay at infinity, then $P_{W}$ is known as the attenuated ray (or Radon) transform on the plane. 
This transform (at least, with $a \geq 0$ ) arises, in particular, in single-photon emission computed tomography (SPECT). Transforms $P_{W}$ with some other weights $W$ also arises in applications. For more information in this connection see, for example, $[\mathrm{Na}],[\mathrm{Ku}],[\mathrm{MP}]$, [Ba2].

Exact and simultaneously explicit inversion formulas for the classical and attenuated Radon transforms on the plane were given for the first time in [Ra] and [No1], respectively. For some other weights $W$, exact and simultaneously explicit inversion formulas were given in $[\mathrm{TM}],[\mathrm{BS}],[\mathrm{G}],[\mathrm{No} 2]$.

For general $P_{W}$, under assumptions (1.2), (1.3), explicit and simultaneously exact (modulo $\operatorname{Ker} P_{W}$ ) inversion formulas seem to be impossible. Nevertheless, due to [BQ], $P_{W}$ is injective on $C_{0}\left(\mathbb{R}^{2}\right)$ (where $C_{0}$ denotes continuous functions with compact support), i.e. $\operatorname{Ker} P_{W}=0$ in $C_{0}\left(\mathbb{R}^{2}\right)$, for real analytic $W$ satisfying (1.3).

Besides, [Bo1] gives an example of infinitely smooth $W$ satisfying (1.2), (1.3) and such that $\operatorname{Ker} P_{W} \neq 0$ in $C_{0}^{\infty}\left(\mathbb{R}^{2}\right)$ (where $C_{0}^{\infty}$ denotes infinitely smooth functions with compact support).

For more information concerning known uniqueness and non-uniqueness results for weighted Radon transforms on the plane see [Q], [Fi], [Ku], [BQ], [Bo1], [Bo2], [No1], [No2] and references therein.

In the present work we develop (exact and approximate) reconstruction methods of $f \in C_{0}\left(\mathbb{R}^{2}\right)$ from $P_{W} f$ with known $W$ satisfying (1.2) and, for example, (1.3). Our studies are based on: (1) the Riemann-Hilbert problem approach of [FN], [No1], [Ba1]; (2) generalized analytic function theory, see [V]; (3) approximate inversion approach of [Ch], [No2]. Some of our results can be considered also as a development of [Ku]. Actually, we consider weights of the form

$$
\begin{aligned}
& W(x, \theta(\varphi))=\sum_{n=-N}^{N} e^{i n \varphi} w_{n}(x), \\
& x \in \mathbb{R}^{2}, \quad \theta(\varphi)=(\cos \varphi, \sin \varphi), \quad \varphi \in[-\pi, \pi], \quad N \in \mathbb{N} \cup 0,
\end{aligned}
$$

where $w_{n} \in C\left(\mathbb{R}^{2}\right) \cap L^{\infty}\left(\mathbb{R}^{2}\right)$ and, for example, $\left|w_{0}\right|>c_{0}>0$. Such weights approximate, in particular, $W$ satisfying (1.2), (1.3).

Due to the formula (see [No2])

$$
\begin{aligned}
& \frac{1}{2}\left(P_{W} f(s, \theta)+P_{W} f(-s,-\theta)\right)=P_{W_{s y m}} f(s, \theta), \quad(s, \theta) \in \mathbb{R} \times \mathbb{S}^{1}, \\
& W_{\text {sym }}(x, \theta)=\frac{1}{2}(W(x, \theta)+W(x,-\theta)), \quad x \in \mathbb{R}^{2}, \quad \theta \in \mathbb{S}^{1},
\end{aligned}
$$

inversion of $P_{W}$ can be reduced to inversion of $P_{W_{s y m}}$. Therefore, in the present work we consider mainly the case when

$$
W(x, \theta)=W(x,-\theta), \quad x \in \mathbb{R}^{2}, \quad \theta \in \mathbb{S}^{1}
$$

and, as a corollary,

$$
w_{n} \equiv 0 \text { on } \mathbb{R}^{2} \text { if } n \text { is odd }
$$


for $W$ of (1.6).

In Theorem 3.1 of Section 3 we reduce inversion of $P_{W}$ under assumptions (1.6), (1.8) to solving a system of first order differential equations on $\mathbb{R}^{2}$ with a decay condition at infinity. In the simplest non-trivial case this system is reduced to the inhomogeneous Beltrami type equation of Remark 3.2 of Section 3.

One of possibilities to obtain a linear integral equation for finding $f$ from $P_{W} f$ and $W$ proceeding from Theorem 3.1 is realized in Theorem 3.2 of Section 3. In addition, the linear integral equation of Theorem 3.2 is uniquely solvable by the method of successive approximations, under some smallness condition on the function set

$\left\{\frac{w_{2 k}}{w_{0}}:|2 k| \leq N, k \neq 0\right\}$. It is interesting to note that for the case when $w_{0} \equiv 1$ the linear integral equation of Theorem 3.2, actually, coincides with related equation of [Ku], obtained in a completely different way.

In turn, proceeding from Theorem 3.2 we obtain, in particular, that if $W$ satisfies (1.2), (1.3) and can be written as

$$
\begin{aligned}
& W(x, \theta(\varphi))=e^{-2 i \varphi} w_{-2}(x)+w_{0}(x)+e^{2 i \varphi} w_{2}(x), \\
& x \in \mathbb{R}^{2}, \quad \theta(\varphi)=(\cos \varphi, \sin \varphi), \quad \varphi \in[-\pi, \pi]
\end{aligned}
$$

then $f \in C_{0}\left(\mathbb{R}^{2}\right)$ is uniquely and efficiently determined by $P_{W} f$ and $W$; see Theorem 3.3 of Section 3 for details.

At least on the theoretical level, our exact inversion method for $P_{W}$, under assumptions (1.2), (1.3), (1.9), and formula (1.7) give new approximate inversion method for $P_{W}$ under general assumptions (1.2), (1.3). For $w_{-2} \equiv 0, w_{2} \equiv 0$ this approximate inversion is reduced to the Chang approximate inversion formula; see, for example, [Ch], [No2], [GN] in connection with Chang's formula.

In studies of the present work we proceed from Proposition 2.1 of Section 2. Due to this proposition we consider inversion of $P_{W}$ as, roughly speaking, some additive RiemannHilbert problem. The main results of the present work on inversion of $P_{W}$ are presented in details in Section 3.

\section{Weighted ray equation}

Assuming that $W$ satisfies $(1.2)$ and $f \in C_{0}\left(\mathbb{R}^{2}\right)$, we consider the equation

$$
\theta \partial_{x} \psi(x, \theta)=W(x, \theta) f(x), \quad x=\left(x_{1}, x_{2}\right) \in \mathbb{R}^{2}, \quad \theta=\left(\theta_{1}, \theta_{2}\right) \in \mathbb{S}^{1},
$$

where $\theta$ is spectral parameter, $\partial_{x}=\left(\partial / \partial x_{1}, \partial / \partial x_{2}\right), \quad \theta \partial_{x}=\sum_{j=1}^{2} \theta_{j} \partial / \partial x_{j}$.

For any $\theta \in \mathbb{S}^{1}$ we consider the continuous solution $\psi^{+}(\cdot, \theta)$ of $(2.1)$, specified by

$$
\lim _{t \rightarrow-\infty} \psi^{+}(x+t \theta, \theta)=0, \quad x \in \mathbb{R}^{2} .
$$

The following formula holds:

$$
\psi^{+}(x, \theta)=\int_{-\infty}^{0} W(x+t \theta, \theta) f(x+t \theta) d t, x \in \mathbb{R}^{2}, \theta \in \mathbb{S}^{1}
$$


In addition,

$$
P_{W} f(s, \theta)=\lim _{t \rightarrow+\infty} \psi^{+}\left(s \theta^{\perp}+t \theta, \theta\right)=0, \quad s \in \mathbb{R}, \quad \theta \in \mathbb{S}^{1},
$$

where $P_{W} f$ is defined by $(1.1), \theta^{\perp}$ is the same that in (1.1).

We say that equation (2.1) is the weighted ray equation on the plane and that $P_{W} f$ is "scattering" data for this equation.

Assuming that $W$ satisfies (1.6) and $f \in C_{0}\left(\mathbb{R}^{2}\right)$ we consider also equation (2.1) with complexified spectral parameter:

$$
\theta \partial_{x} \psi(x, \theta)=W(x, \theta) f(x), \quad x \in \mathbb{R}^{2}, \quad \theta \in \Sigma,
$$

where

$$
\Sigma=\left\{\theta=\left(\theta_{1}, \theta_{2}\right) \in \mathbb{C}^{2}: \theta_{1}^{2}+\theta_{2}^{2}=1\right\}
$$

For any $\theta \in \Sigma \backslash \mathbb{S}^{1}$ we consider the continuous solution $\psi(\cdot, \theta)$ of $(2.5)$ specified by

$$
\psi(x, \theta) \rightarrow 0 \text { as }|x| \rightarrow \infty
$$

The following formula holds:

$$
\psi(x, \theta)=\int_{\mathbb{R}^{2}} G(x-y, \theta) W(y, \theta) f(y) d y, \quad x \in \mathbb{R}^{2}, \theta \in \Sigma \backslash \mathbb{S}^{1},
$$

where

$$
G(x, \theta)=\frac{\operatorname{sgn}\left(\operatorname{Re} \theta_{1} \operatorname{Im} \theta_{2}-\operatorname{Re} \theta_{2} \operatorname{Im} \theta_{1}\right)}{-2 \pi i\left(\theta_{2} x_{1}-\theta_{1} x_{2}\right)},
$$

$W$ is defined by (1.6) with $e^{i n \varphi}$ replaced by $\left(\theta_{1}+i \theta_{2}\right)^{n}$.

$$
\theta \partial_{x} G(x, \theta)=\delta(x), \quad x \in \mathbb{R}^{2}, \quad \theta \in \Sigma \backslash \mathbb{S}^{1} .
$$

For detail properties of $G(x, \theta)$ we refer to [No1].

Consider

$$
z=x_{1}+i x_{2}, \quad \bar{z}=x_{1}-i x_{2}, x=\left(x_{1}, x_{2}\right) \in \mathbb{R}^{2} .
$$

Consider the following parametrization of $\Sigma$ by $\lambda \in \mathbb{C} \backslash 0$ :

$$
\begin{aligned}
& \lambda(\theta)=\theta_{1}+i \theta_{2} \text { for } \theta=\left(\theta_{1}, \theta_{2}\right) \in \Sigma \\
& \theta_{1}(\lambda)=\frac{1}{2}\left(\lambda+\frac{1}{\lambda}\right), \quad \theta_{2}(\lambda)=\frac{1}{2 i}\left(\lambda-\frac{1}{\lambda}\right) \text { for } \lambda \in \mathbb{C} \backslash 0 .
\end{aligned}
$$

In the variable $\lambda$ the circle $\mathbb{S}^{1}=\Sigma \cap \mathbb{R}^{2}$ takes the form

$$
\mathbb{T}=\{\lambda \in \mathbb{C}: \quad|\lambda|=1\}
$$


Let $\psi(z, \lambda), G(z, \lambda)$ denote $\psi$ and $G$ of (2.8), (2.9) written in the variables $z, \lambda$ of (2.11), (2.12). Let $W(z, \lambda), f(z)$ denote related $W$ and $f$ written in the same way. We recall that

$$
G(z, \lambda)=\frac{\operatorname{sgn}(|\lambda|-1)}{\pi(\lambda \bar{z}-z / \lambda)}, \quad z \in \mathbb{C}, \lambda \in \mathbb{C} \backslash(\mathbb{T} \cup 0)
$$

see [No1], [Ba1] for detail properties of $G$. Note also that, under assumptions (1.6), (1.8), $W$ can be written as

$$
W(z, \lambda)=\sum_{l=-m}^{m} \lambda^{2 l} w_{2 l}(z), \quad \lambda \in \mathbb{C} \backslash 0, z \in \mathbb{C},
$$

where $w_{2 l} \in C(\mathbb{C}) \cap L^{\infty}(\mathbb{C}), m=[N / 2]$ (i.e. $m$ is the integer part of $N / 2$ ). In addition, we have the following proposition:

Proposition 2.1. Let $\psi$ be the function of (2.8), where $W$ satisfies (1.6), (1.8), $[N / 2]=m$, and $f \in C_{0}\left(\mathbb{R}^{2}\right)$. Then for each $z \in \mathbb{C}$ the function $\psi=\psi(z, \lambda)$ has the following properties in $\lambda$ (in variables $z, \lambda$ of (2.11), (2.12)):

$$
\begin{gathered}
\frac{\partial}{\partial \bar{\lambda}} \psi(z, \lambda)=0 \text { for } \lambda \in \mathbb{C} \backslash(\mathbb{T} \cup 0) ; \\
\psi(z, \lambda)=\sum_{j=0}^{m} \lambda^{2 j-1} u_{2 j-1}^{-}(z)+O\left(\lambda^{-3}\right), \lambda \rightarrow \infty, \\
u_{2 j-1}^{-}(z)=\frac{1}{\pi} \sum_{k=0}^{m-j} \int_{\mathbb{C}} \frac{(z-\zeta)^{k}}{(\bar{z}-\bar{\zeta})^{k+1}} w_{2(k+j)}(\zeta) f(\zeta) d \operatorname{Re} \zeta d \operatorname{Im} \zeta, \\
\psi(z, \lambda)=\sum_{j=0}^{m} \lambda^{1-2 j} u_{1-2 j}^{+}(z)+O\left(\lambda^{3}\right), \lambda \rightarrow 0, \\
u_{1-2 j}^{+}(z)=\frac{1}{\pi} \sum_{k=0}^{m-j} \int_{\mathbb{C}} \frac{(\bar{z}-\bar{\zeta})^{k}}{(z-\zeta)^{k+1}} w_{-2(k+j)}(\zeta) f(\zeta) d \operatorname{Re} \zeta d \operatorname{Im} \zeta ; \\
\psi_{+}(z, \lambda)-\psi_{-}(z, \lambda)=\varphi(z, \lambda), \quad \lambda \in \mathbb{T},
\end{gathered}
$$

where

$$
\begin{aligned}
& \psi_{ \pm}(z, \lambda)=\psi(z, \lambda(1 \mp 0)), \quad \lambda \in \mathbb{T} \\
& \varphi(z, \lambda)=\frac{1}{\pi i} p \cdot v \cdot \int_{\mathbb{R}} \frac{P_{W} f(s, \theta(\lambda))}{x(z) \theta^{\perp}(\lambda)-s} d s, \lambda \in \mathbb{T},
\end{aligned}
$$

where $x(z)=\left(x_{1}(z), x_{2}(z)\right), \theta(\lambda)=\left(\theta_{1}(\lambda), \theta_{2}(\lambda)\right)$ are given according to (2.11), (2.12), $\theta^{\perp}=\left(-\theta_{2}, \theta_{1}\right)$ for $\theta=\left(\theta_{1}, \theta_{2}\right)$. 
Proposition 2.1 follows from considerations given in [No1], [Ba1]. In particulier, for $[N / 2]=m=0$ Proposition 2.1 is a particular case of results of [No1] (and goes back to $[\mathrm{FN}])$.

Properties (2.16)-(2.21) of $\psi$ permit to study inversion of $P_{W}$ by means of additive Riemann-Hilbert problem techniques.

3. Inversion of $P_{W}$

One can see that

$$
\begin{aligned}
& P_{W} f=P_{\tilde{W}}\left(w_{0} f\right), \\
& \tilde{W}(x, \theta)=W(x, \theta) / w_{0}(x), \quad x \in \mathbb{R}^{2}, \theta \in \mathbb{S}^{1},
\end{aligned}
$$

under the assumption that

$$
\left|w_{0}\right|>c_{0}>0
$$

where

$$
w_{0}(x)=\frac{1}{2 \pi} \int_{\mathbb{S}^{1}} W(x, \theta) d \theta, \quad x \in \mathbb{R}^{2} .
$$

Therefore, under assumptions (1.2), (3.3), inversion of $P_{W}$ is reduced to the case when $w_{0} \equiv 1$ on $\mathbb{R}^{2}$.

Let

$$
\begin{aligned}
& f_{\text {appr }}(x)=F_{0}(x) / w_{0}(x), \\
& F_{0}(x)=\frac{1}{4 \pi} \int_{\mathbb{S}^{1}} h^{\prime}\left(x \theta^{\perp}, \theta\right) d \theta, \quad h^{\prime}(s, \theta)=\frac{d}{d s} h(s, \theta), \\
& h(s, \theta)=\frac{1}{\pi} p \cdot v \cdot \int_{\mathbb{R}} \frac{P_{W} f(t, \theta)}{s-t} d t, \quad s \in \mathbb{R}, \theta \in \mathbb{S}^{1}, x \in \mathbb{R}^{2} .
\end{aligned}
$$

We recall that (3.5) is known as the Chang approximate inversion formula for $P_{W}$ under assumptions (1.2), (3.3). In addition, under assumptions (1.2), (3.3), due to Theorem 1 of [No2], we have that

$$
f_{\text {appr }}=f \quad \text { (in the sense of distributions) on } \mathbb{R}^{2} \text { for all } f \in C_{0}\left(\mathbb{R}^{2}\right),
$$

if and only if

$$
W(x, \theta)-w_{0}(x)=w_{0}(x)-W(x,-\theta), \quad x \in \mathbb{R}^{2}, \theta \in \mathbb{S}^{1}
$$

However, property (3.7) is fulfilled for $W$ of the form (1.6) if and only if

$$
\sum_{l=1}^{[N / 2]}\left(\left|w_{2 l}\right|+\left|w_{-2 l}\right|\right) \equiv 0 \text { on } \mathbb{R}^{2}
$$


Below in this section we are focused on inversion of $P_{W}$ under assumptions (1.2), (1.8), (3.3) for the case when (3.8) is not necessary fulfilled. In addition, we assume that $W$ is written in the form $(2.15)$, where $m \geq 1$.

Consider the $2 m \times 2 m$ operator matrices $A$ and $B$ such that

$$
\begin{gathered}
A_{j, j}=\partial_{z}, \quad j=1, \ldots, m, \\
A_{j+1, j}=\partial_{\bar{z}}, \quad j=1, \ldots, m-1, \\
A_{j, j}=\partial_{\bar{z}}, \quad j=m+1, \ldots, 2 m, \\
A_{j, j+1}=\partial_{z}, \quad j=m+1, \ldots, 2 m-1, \\
\quad A_{i, j}=0 \text { for all other cases, } \\
B_{j, m}=\tilde{w}_{2(m-j+1)}(z) \partial_{\bar{z}}, \quad j=1, \ldots, m, \\
B_{j, m}=\tilde{w}_{-2(j-m)}(z) \partial_{\bar{z}}, \quad j=m+1, \ldots, 2 m, \\
B_{j, m+1}=\tilde{w}_{2(m-j+1)}(z) \partial_{z}, \quad j=1, \ldots, m, \\
B_{j, m+1}=\tilde{w}_{-2(j-m)}(z) \partial_{z}, \quad j=m+1, \ldots, 2 m, \\
B_{i, j}=0 \text { for all other cases, }
\end{gathered}
$$

where

$$
\begin{aligned}
& \partial_{z}=\frac{1}{2}\left(\frac{\partial}{\partial x_{1}}-i \frac{\partial}{\partial x_{2}}\right), \quad \partial_{\bar{z}}=\frac{1}{2}\left(\frac{\partial}{\partial x_{1}}+i \frac{\partial}{\partial x_{2}}\right), \\
& \tilde{w}_{2 l}=w_{2 l} / w_{0}, \quad \tilde{w}_{-2 l}=w_{-2 l} / w_{0}, \quad l=0,1, \ldots, m .
\end{aligned}
$$

Consider the vector-functions $q=\left(q_{1}, \ldots, q_{2 m}\right), v=\left(v_{1}, \ldots, v_{2 m}\right)$ such that

$$
\begin{gathered}
q_{j}=\tilde{w}_{2(m-j+1)}(z) F_{0}(z), \quad j=1, \ldots, m, \\
q_{j}=\tilde{w}_{-2(j-m)}(z) F_{0}(z), \quad j=m+1, \ldots, 2 m, \\
v_{j}=u_{2(m-j+1)-1}^{-}(z), \quad j=1, \ldots, m, \\
v_{j}=u_{-2(j-m)+1}^{+}(z), \quad j=m+1, \ldots, 2 m,
\end{gathered}
$$

where $F_{0}$ is the function of $(3.5), \tilde{w}_{ \pm 2 l}$ are defined by $(3.12), u_{2 l-1}^{-}, u_{-2 l+1}^{+}$are the function of $(2.17),(2.18), l=1, \ldots, m$.

Let $\chi_{D}$ denote the characteristic function of a domain $D$ in $\mathbb{C}$, i.e.

$$
\chi_{D} \equiv 1 \text { on } D, \chi_{D} \equiv 0 \text { on } \mathbb{C} \backslash D .
$$

Let

$$
\begin{aligned}
& v^{\prime}(z)=\left(\partial_{z} v_{1}(z), \ldots, \partial_{z} v_{m}(z), \quad \partial_{\bar{z}} v_{m+1}(z), \ldots, \partial_{\bar{z}} v_{2 m}(z)\right) \\
& \text { for } v(z)=\left(v_{1}(z), \ldots, v_{2 m}(z)\right), \quad z \in \mathbb{C} .
\end{aligned}
$$

Theorem 3.1. Let $W$ satisfy (2.15), (3.3) and $f \in C(\mathbb{C})$, supp $f \subset D$, where $m \geq 1$ and $D$ is an open bounded domain in $\mathbb{C}$. Then:

$$
\begin{aligned}
& \left(A-\chi_{D} B\right) v=\chi_{D} q, \\
& v \in C(\mathbb{C}), \quad v^{\prime} \in L^{2}(\mathbb{C}), \quad|v(z)| \rightarrow 0 \text { as }|z| \rightarrow \infty,
\end{aligned}
$$


where $A, B, q, v, \chi_{D}$ are defined by (3.9), (3.10), (3.13), (3.14), (3.15) and $v^{\prime}$ is defined by (3.16) in terms of $v$. In addition,

$$
f(z)=\frac{F_{0}(z)+\partial_{\bar{z}} v_{m}(z)+\partial_{z} v_{m+1}(z)}{w_{0}(z)}, \quad z \in \mathbb{C},
$$

where $F_{0} / w_{0}$ is the function of (3.5) and $v_{m}, v_{m+1}$ are the components of $v$.

We consider (3.17), (3.18) as a system of first order differential equations for $v=\left(v_{1}, \ldots, v_{2 m}\right)$ on $\mathbb{C}$ with a decay condition at infinity. One can see that $: A$ is independent of $W$ and $F_{0}, B$ is explicitely given in terms of $W$ and is independent of $F_{0}$, $q$ is explicitely given in terms of $W$ and $F_{0}$. In addition, $f$ can be found via (3.19) as soon as $F_{0} / w_{0}, v_{m}, v_{m+1}$ are known.

Theorem 3.1 is proved in Section 4 .

Lemma 3.1. Let the assumptions of Theorem 3.1 be fulfilled and

$$
\begin{aligned}
& \sum_{l=1}^{m} \sup _{z \in D}\left|\tilde{w}_{2 l}(z)\right|<\frac{1}{2}, \\
& \sum_{l=1}^{m} \sup _{z \in D}\left|\tilde{w}_{-2 l}(z)\right|<\frac{1}{2} .
\end{aligned}
$$

Then system (3.16), (3.17) is uniquely solvable for $v$.

Lemma 3.1 is proved in Section 5.

Theorem 3.1 and Lemma 3.1 imply, in particular, that $P_{W}$ is injective on

$$
C_{D}(\mathbb{C})=\{f \in C(\mathbb{C}): \quad \operatorname{supp} f \subset D\},
$$

under assumptions (2.15), (3.3), (3.20), where $D$ is an open bounded domain in $\mathbb{C}$.

Remark 3.1. If $W$ of (2.15) is real-valued for $\lambda \in \mathbb{T}, z \in \mathbb{C}$, then

$$
w_{-2 l}=\bar{w}_{2 l} \text { on } \mathbb{C}, \quad l=0,1, \ldots, m
$$

In this case both inequalities (3.20) are reduced to each of them. If, in addition, $f$ is continuous compactly supported and also real-valued, then

$$
v_{2 m-j+1}=\bar{v}_{j} \text { on } \mathbb{C}, \quad j=1, \ldots, m,
$$

where $v=\left(v_{1}, \ldots, v_{2 m}\right)$ is defined by means of (3.14), (2.17), (2.18).

Remark 3.2. The system (3.17), (3.18) with symmetries (3.22), (3.23) and with $m=1$ is reduced to the following scalar equation

$$
\begin{aligned}
& \partial_{z} u-2 \chi_{D} \tilde{w}_{2} \operatorname{Re} \partial_{\bar{z}} u=\chi_{D} \tilde{w}_{2} F_{0}, \\
& u \in C(\mathbb{C}), \quad \partial_{z} u \in L^{2}(\mathbb{C}), \quad|u(z)| \rightarrow 0, \quad \text { as }|z| \rightarrow \infty,
\end{aligned}
$$


where $u=v_{1}, \bar{u}=v_{2}$. In addition, formula (3.19) is reduced to the formula

$$
f=\frac{F_{0}+2 R e \partial_{\bar{z}} u}{w_{0}} .
$$

There are different possibilities to obtain a linear integral equation for finding $f$ from $F_{0}$ and $W$ proceeding from Theorem 3.1. One of these possibilities is realized as equation (5.11) of Section 5. Another one is realized as Theorem 3.2 given below.

Let $T, \Pi, \bar{T}, \bar{\Pi}$ denote the linear integral operators on the complex plane such that

$$
\begin{aligned}
& T u(z)=-\frac{1}{\pi} \int_{\mathbb{C}} \frac{u(\zeta)}{\zeta-z} d \operatorname{Re} \zeta d \operatorname{Im} \zeta, \\
& \Pi u(z)=\partial_{z} T u(z)=-\frac{1}{\pi} \int_{\mathbb{C}} \frac{u(\zeta)}{(\zeta-z)^{2}} d \operatorname{Re} \zeta d \operatorname{Im} \zeta, \\
& \bar{T} u(z)=-\frac{1}{\pi} \int_{\mathbb{C}} \frac{u(\zeta)}{\bar{\zeta}-\bar{z}} d \operatorname{Re} \zeta d \operatorname{Im} \zeta, \\
& \bar{\Pi} u(z)=\partial_{\bar{z}} T u(z)=-\frac{1}{\pi} \int_{\mathbb{C}} \frac{u(\zeta)}{(\bar{\zeta}-\bar{z})^{2}} d \operatorname{Re} \zeta d \operatorname{Im} \zeta,
\end{aligned}
$$

where $u$ is a test function, $z \in \mathbb{C}$; see, for example, $[\mathrm{V}]$ for detail properties of these operators.

Theorem 3.2. Let $W$ satisfy (2.15), (3.3) (where $m \geq 1$ ). Let $F=w_{0} f, f \in C(\mathbb{C}$ ), supp $f \subset D$, where $D$ is an open bounded domain in $\mathbb{C}$. Then

$$
F+\sum_{l=1}^{m}\left((-\bar{\Pi})^{l} \tilde{w}_{2 l}+(-\Pi)^{l} \tilde{w}_{-2 l}\right) \chi_{D} F=F_{0},
$$

where $\tilde{w}_{ \pm 2 l}, \chi_{D}$ are the multiplication operators by the function of (3.12), (3.15), П, $\bar{\Pi}$ are the operators of (3.27), (3.28), $F_{0}$ is the function of (3.5). In addition, equation (3.29) is uniquely solvable for $F$ in $L^{2}(\mathbb{C})$ (by the method of successive approximations) if

$$
\sum_{l=1}^{m}\left(\sup _{z \in D}\left|\tilde{w}_{2 l}(z)\right|+\sup _{z \in D}\left|\tilde{w}_{-2 l}(z)\right|\right)<1 .
$$

Theorem 3.2 is proved in Section 6.

One can show that for the case when $w_{0} \equiv 1$ equation (3.29), actually, coincides with related equation of $[\mathrm{Ku}]$. In $[\mathrm{Ku}]$ it was shown that this equation of $[\mathrm{Ku}]$ is uniquely solvable if

$$
\frac{\sum_{l=1}^{m}\left(\sup _{z \in D}\left|\tilde{w}_{2 l}(z)\right|+\sup _{z \in D}\left|\tilde{w}_{-2 l}(z)\right|\right)}{\min _{z \in D}\left|w_{0}(z)\right|}<1 .
$$


One can see that condition (3.30) is considerably less restrictive than (3.31), in general.

In addition, for $m=1$ one can show that if $W$ is given by (2.15) and

$$
\overline{W(z, \lambda)}=W(z, \lambda) \geq c>0, z \in D, \lambda \in \mathbb{T}
$$

then

$$
\left|w_{0}(z)\right|-\left|w_{-2}(z)\right|-\left|w_{2}(z)\right| \geq c, \quad z \in D,
$$

and condition (3.30) is fulfilled. Therefore, proceeding from Theorem 3.2 we have also, in particular, the following result:

Theorem 3.3. Let $W$ be given by (2.15) for $m=1$ and conditions (3.32) be fulfilled, and let $f \in C(\mathbb{C})$, supp $f \subset D$, where $D$ is an open bounded domain in $\mathbb{C}$. Then $f$ is uniquely determined by $P_{W} f$ and $W$ via the linear integral equation (3.29) for $m=1$ (solvable by the method of successive approximations in $L^{2}(\mathbb{C})$ ).

Note that Theorems 3.2 and 3.3 remain valid under assumptions that $f \in L^{2}(D)$, $f \equiv 0$ on $\mathbb{C} \backslash D$.

\section{Proof of Theorem 3.1}

Note that

$$
P_{W} f(s, \theta)=P_{W} f(-s,-\theta), \quad(s, \theta) \in \mathbb{R} \times \mathbb{S}^{1} .
$$

As a corollary, we have that

$$
\varphi(z,-\lambda)=-\varphi(z, \lambda), z \in \mathbb{C}, \lambda \in \mathbb{T},
$$

where $\varphi$ is defined by $(2.21)$.

Let

$$
\psi^{0}(z, \lambda)=\frac{1}{2 \pi i} \int_{\mathbb{T}} \frac{\varphi(z, \zeta)}{\zeta-\lambda} d \zeta, z \in \mathbb{C}, \lambda \in \mathbb{C} \backslash \mathbb{T},
$$

where $\varphi$ is defined by (2.21). Then for each $z \in \mathbb{C}$ the function $\psi^{0}=\psi^{0}(z, \lambda)$ has the following properties in $\lambda$ :

$$
\begin{aligned}
& \frac{\partial}{\partial \bar{\lambda}} \psi^{0}(z, \lambda)=0 \text { for } \lambda \in \mathbb{C} \backslash \mathbb{T}, \\
& \psi^{0}(z, \lambda)=\lambda^{-1} \psi_{-1}^{0}(z)+O\left(\lambda^{-3}\right), \lambda \rightarrow \infty, \\
& \psi^{0}(z, \lambda)=\lambda \psi_{1}^{0}(z)+O\left(\lambda^{3}\right), \lambda \rightarrow 0, \\
& \psi_{+}^{0}(z, \lambda)-\psi_{-}^{0}(z, \lambda)=\varphi(z, \lambda), \lambda \in \mathbb{T},
\end{aligned}
$$

where

$$
\psi_{ \pm}^{0}(z, \lambda)=\psi(z, \lambda(1 \mp 0)), \quad \lambda \in \mathbb{T},
$$

and also

$$
\begin{aligned}
& \psi_{-1}^{0}(z)=-\frac{1}{2 \pi i} \int_{\mathbb{T}} \varphi(z, \zeta) d \zeta \\
& \psi_{1}^{0}(z)=\frac{1}{2 \pi i} \int_{\mathbb{T}} \frac{\varphi(z, \zeta)}{\zeta^{2}} d \zeta .
\end{aligned}
$$


Properties (4.4)-(4.10) follow from (4.3), where to obtain (4.5), (4.6) we use also (4.2). Let

$$
\begin{aligned}
& \mu(z, \lambda)=\psi(z, \lambda)-\psi^{0}(z, \lambda)-\sum_{j=1}^{m} \lambda^{2 j-1} u_{2 j-1}^{-}(z)-\sum_{j=1}^{m} \lambda^{1-2 j} u_{1-2 j}^{+}(z), \\
& z \in \mathbb{C}, \quad \lambda \in \mathbb{C} \backslash(\mathbb{T} \cup 0),
\end{aligned}
$$

where $\psi$ is the function of Proposition $2.1, \psi^{0}$ is defined by $(4.3), u_{2 j-1}^{-}, u_{1-2 j}^{+}$are the coefficients of formulas (2.17), (2.18). Then for each $z \in \mathbb{C}$ the function $\mu=\mu(z, \lambda)$ has the following properties in $\lambda$ :

$$
\begin{aligned}
& \frac{\partial}{\partial \bar{\lambda}} \mu(z, \lambda)=0 \text { for } \lambda \in \mathbb{C} \backslash(\mathbb{T} \cup 0), \\
& \mu(z, \lambda) \rightarrow 0, \quad \lambda \rightarrow \infty \\
& \mu(z, \lambda) \rightarrow 0, \quad \lambda \rightarrow 0, \\
& \mu_{+}(z, \lambda)-\mu_{-}(z, \lambda)=0, \quad \lambda \in \mathbb{T}
\end{aligned}
$$

where

$$
\mu_{ \pm}(z, \lambda)=\mu(z, \lambda(1 \mp 0)), \quad \lambda \in \mathbb{T} .
$$

Properties (4.12)-(4.16) follow from (2.16)-(2.20), (4.4)-(4.8) and definition (4.11). Using (4.12)-(4.16) we obtain that

$$
\mu(z, \lambda) \equiv 0, \quad z \in \mathbb{C}, \quad \lambda \in \mathbb{C} .
$$

Due to (4.11), (4.17) we have that

$$
\begin{aligned}
& \psi(z, \lambda)=\sum_{j=1}^{m} \lambda^{2 j-1} u_{2 j-1}^{-}(z)+\sum_{j=1}^{m} \lambda^{1-2 j} u_{1-2 j}^{+}(z)+\psi^{0}(z, \lambda), \\
& z \in \mathbb{C}, \quad \lambda \in \mathbb{C} \backslash(\mathbb{T} \cup 0) .
\end{aligned}
$$

Due to equation (2.5) for $\psi$ we have that

$$
\left(\lambda \partial_{z}+\lambda^{-1} \partial_{\bar{z}}\right) \psi(z, \lambda)=W(z, \lambda) f(z), \quad \lambda \in \mathbb{C} \backslash(\mathbb{T} \cup 0), \quad z \in \mathbb{C},
$$

where $W$ is given by (2.15) and $\psi$ is written according to (4.18). Proceeding from (2.15), (4.5), (4.6), (4.18), (4.19) we obtain that

$$
\begin{aligned}
& \sum_{j=1}^{m} \lambda^{2 j} \partial_{z} u_{2 j-1}^{-}(z)+\sum_{j=0}^{m-1} \lambda^{2 j} \partial_{\bar{z}} u_{2 j+1}^{-}(z)+\partial_{z} u_{-1}^{+}(z)+\partial_{z} \psi_{-1}^{0}(z)+o(1)= \\
& \sum_{j=0}^{m} \lambda^{2 j} w_{2 j}(z) f(z) \text { for } \lambda \rightarrow \infty, \quad z \in \mathbb{C}
\end{aligned}
$$




$$
\begin{aligned}
& \sum_{j=1}^{m} \lambda^{-2 j} \partial_{\bar{z}} u_{1-2 j}^{+}(z)+\sum_{j=0}^{m-1} \lambda^{-2 j} \partial_{z} u_{-1-2 j}^{+}(z)+\partial_{\bar{z}} u_{1}^{-}(z)+\partial_{\bar{z}} \psi_{1}^{0}(z)+o(1)= \\
& \sum_{j=-m}^{-1} \lambda^{2 j} \partial_{\bar{z}} u_{2 j+1}^{+}(z)+\sum_{j=-m+1}^{0} \lambda^{2 j} \partial_{z} u_{2 j-1}^{+}(z)+\partial_{\bar{z}} u_{1}^{-}(z)+\partial_{\bar{z}} \psi_{1}^{0}(z)+o(1)= \\
& \sum_{j=-m}^{0} \lambda^{2 j} w_{2 j}(z) f(z) \text { for } \lambda \rightarrow 0, \quad z \in \mathbb{C} .
\end{aligned}
$$

In addition, proceeding from $(2.21),(3.11),(4.9),(4.10)$ one can see that

$$
\begin{aligned}
& \partial_{z} \psi_{-1}^{0}(z)=F_{0}(z), \quad z \in \mathbb{C}, \\
& \partial_{\bar{z}} \psi_{1}^{0}(z)=F_{0}(z), \quad z \in \mathbb{C},
\end{aligned}
$$

where $F_{0}$ is the function of (3.5).

Formulas (4.20), (4.21) imply that:

$$
\begin{aligned}
& \partial_{z} u_{2 j-1}^{-}(z)=w_{2 j}(z) f(z) \text { for } j=m, \\
& \partial_{z} u_{2 j-1}^{-}(z)+\partial_{\bar{z}} u_{2 j+1}^{-}(z)=w_{2 j}(z) f(z) \text { for } j=1, \ldots, m-1, \\
& \partial_{z} u_{-1}^{+}(z)+\partial_{\bar{z}} u_{1}^{-}(z)+F_{0}(z)=w_{0}(z) f(z) ; \\
& \partial_{\bar{z}} u_{2 j+1}^{+}(z)=w_{2 j}(z) f(z) \text { for } j=-m, \\
& \partial_{\bar{z}} u_{2 j+1}^{+}(z)+\partial_{z} u_{2 j-1}^{+}(z)=w_{2 j}(z) f(z) \text { for } j=-m+1, \ldots,-1, \\
& \partial_{\bar{z}} u_{1}^{-}(z)+\partial_{z} u_{-1}^{+}(z)+F_{0}(z)=w_{0}(z) f(z),
\end{aligned}
$$

where $z \in \mathbb{C}$. In turn, formulas $(4.22)$ can be rewritten as

$$
\begin{aligned}
& A v=f \omega, \\
& \partial_{\bar{z}} v_{m}+\partial_{z} v_{m+1}+F_{0}=w_{0} f,
\end{aligned}
$$

where $A$ is the operator matrix defined by (3.9), $v$ is the vector-function defined by (3.14), $\omega=\left(\omega_{1}, \ldots, \omega_{2 m}\right)$ is the vector-function such that

$$
\begin{aligned}
& \omega_{j}=w_{2(m-j+1)}, \quad j=1, \ldots, m, \\
& \omega_{j}=w_{-2(j-m)}, \quad j=m+1, \ldots, 2 m,
\end{aligned}
$$

and $v_{m}, v_{m+1}$ are the components of $v$.

In addition, we have that

$$
f=\chi_{D} f
$$

Formulas (4.23), (4.26) imply that

$$
A v=\chi_{D} f \omega
$$


Formulas (3.3), (4.24), (4.27) imply that

$$
A v=\chi_{D}\left(\frac{\partial_{\bar{z}} v_{m}+\partial_{z} v_{m+1}}{w_{0}}\right) \omega
$$

One can see that, under assumption (3.3), formula (3.17) is equivalent to (4.28) and formula (3.19) is equivalent to (4.24).

Under our assumptions, the properties that $v \in C(\mathbb{C}),|v(z)| \rightarrow 0$ as $|z| \rightarrow \infty$ follow form formulas for $u_{2 j-1}^{-}$and $u_{1-2 j}^{+}$of (2.17), (2.18). Under our assumptions, the property that $v^{\prime} \in L^{2}(\mathbb{C})$ can be obtained recurrently for its components $v_{1}^{\prime}, \ldots, v_{m}^{\prime}$ and for its components $v_{2 m}^{\prime}, \ldots, v_{m+1}^{\prime}$ proceeding from definition (3.16), equation (4.27) and the result that (see $[\mathrm{V}]$ )

$$
\|\Pi\|_{L^{2}(\mathbb{C}) \rightarrow L^{2}(\mathbb{C})}=1, \quad\|\bar{\Pi}\|_{L^{2}(\mathbb{C}) \rightarrow L^{2}(\mathbb{C})}=1,
$$

where $\Pi, \bar{\Pi}$ are the operators of $(3.27)$, (3.28).

This completes the proof of Theorem 3.1.

\section{Proof of Lemma 3.1}

Let $\hat{A}, \hat{B}, \Theta$ denote the $2 m \times 2 m$ operator matrices such that

$$
\begin{gathered}
\hat{A}_{j, j}=1, \quad j=1, \ldots, 2 m, \\
\hat{A}_{j+1, j}=\bar{\Pi}, \quad j=1, \ldots, m-1, \\
\hat{A}_{j, j+1}=\Pi, \quad j=m+1, \ldots, 2 m, \\
\hat{A}_{i, j}=0 \text { for all other cases, } \\
\hat{B}_{j, m}=\tilde{w}_{2(m-j+1)} \bar{\Pi}, \quad j=1, \ldots, m, \\
\hat{B}_{j, m}=\tilde{w}_{-2(j-m)} \bar{\Pi}, \quad j=m+1, \ldots, 2 m, \\
\hat{B}_{j, m+1}=\tilde{w}_{2(m-j+1)} \Pi, \quad j=1, \ldots, m, \\
\hat{B}_{j, m+1}=\tilde{w}_{-2(j-m)} \Pi, \quad j=m+1, \ldots, 2 m, \\
\hat{B}_{i, j}=0 \text { for all other cases, } \\
\Theta_{i, j}=(-\bar{\Pi})^{i-j}, \quad 1 \leq j \leq i \leq m, \\
\Theta_{i, j}=(-\Pi)^{j-i}, \quad m+1 \leq i \leq j \leq 2 m, \\
\Theta_{i, j}=0 \text { for all other cases, }
\end{gathered}
$$

where $\Pi, \bar{\Pi}$ are the operators of (3.27), (3.28), $\tilde{w}_{ \pm 2 l}$ are the functions of (3.12).

In addition to (4.29), we recall that (see [Ri], [V] and references therein)

$$
\|\Pi\|_{L^{p}(\mathbb{C}) \rightarrow L^{p}(\mathbb{C})}=\|\bar{\Pi}\|_{L^{p}(\mathbb{C}) \rightarrow L^{p}(\mathbb{C})}=\lambda_{p}, \quad p>1,
$$

where for any $\varepsilon>0$ there is $\delta(\varepsilon)>0$ such that

$$
\lambda_{p}<1+\varepsilon \text { if }|p-2|<\delta(\varepsilon) .
$$


One can see that in terms of $v^{\prime}$ defined by (3.16) system (3.17), (3.18) takes the form

$$
\left(\hat{A}-\chi_{D} \hat{B}\right) v^{\prime}=\chi_{D} q, \quad v^{\prime} \in L^{2}(\mathbb{C}) .
$$

It is necessary to note that, under our assumptions, we have that

$$
\chi_{D} q \in L^{p}(\mathbb{C}), \quad p>1 .
$$

Property (5.6) follows from (5.5) and the property that $v^{\prime} \in L^{p}(\mathbb{C}), p>1$, where $v^{\prime}$ is defined as in Theorem 3.1. In turn, the property that $v^{\prime} \in L^{p}(\mathbb{C}), p>1$, is proved in a similar way as for $p=2$ (using (5.4a) instead of (4.29)).

Besides, one can see that

$$
\Theta A=I, \quad A \Theta=I,
$$

where $I$ is the identity operator matrix.

As a corollary of (5.7), system (5.5) is equivalent to the system

$$
\left(I-\Theta \chi_{D} \hat{B}\right) v^{\prime}=\Theta \chi_{D} q, \quad v^{\prime} \in L^{2}(\mathbb{C}) .
$$

In addition, formulas (5.2), (5.3) imply that

$$
\begin{aligned}
& \left(\Theta \chi_{D} \hat{B}\right)_{i, j}=0 \text { if } j \notin\{m, m+1\}, \\
& r_{1,1} \stackrel{\text { def }}{=}\left(\Theta \chi_{D} \hat{B}\right)_{m, m}=\sum_{j=1}^{m}(-\bar{\Pi})^{m-j} \chi_{D} \tilde{w}_{2(m-j+1)} \bar{\Pi} \\
& r_{1,2} \stackrel{\text { def }}{=}\left(\Theta \chi_{D} \hat{B}\right)_{m, m+1}=\sum_{j=1}^{m}(-\bar{\Pi})^{m-j} \chi_{D} \tilde{w}_{2(m-j+1)} \Pi, \\
& r_{2,1} \stackrel{\text { def }}{=}\left(\Theta \chi_{D} \hat{B}\right)_{m+1, m}=\sum_{j=m+1}^{2 m}(-\Pi)^{j-m-1} \chi_{D} \tilde{w}_{-2(j-m)} \bar{\Pi}, \\
& r_{2,2} \stackrel{\text { def }}{=}\left(\Theta \chi_{D} \hat{B}\right)_{m+1, m+1}=\sum_{j=m+1}^{2 m}(-\Pi)^{j-m-1} \chi_{D} \tilde{w}_{-2(j-m)} \Pi .
\end{aligned}
$$

Let

$$
g_{1}=\left(\Theta \chi_{D} q\right)_{m}, \quad g_{2}=\left(\Theta \chi_{D} q\right)_{m+1},
$$

where $\Theta \chi_{D} q$ is the vector-function of the right side of (5.8).

Using (5.9) we obtain that system (5.8) is reduced to the system

$$
\left(\left(\begin{array}{ll}
1 & 0 \\
0 & 1
\end{array}\right)-\left(\begin{array}{ll}
r_{11} & r_{12} \\
r_{21} & r_{22}
\end{array}\right)\right)\left(\begin{array}{l}
v_{m}^{\prime} \\
v_{m+1}^{\prime}
\end{array}\right)=\left(\begin{array}{l}
g_{1} \\
g_{2}
\end{array}\right)
$$

where $r_{i, j}$ are defined in (5.9), $g_{j}$ are defined by (5.10), and to the formula

$$
v^{\prime}=\Theta \chi_{D} \hat{B} \chi_{\{m, m+1\}} v^{\prime}+\Theta \chi_{D} q
$$


where $\chi_{\{m, m+1\}} v^{\prime}$ denotes the vector-function of the length $2 m$ such that

$$
\begin{aligned}
& \left(\chi_{\{m, m+1\}} v^{\prime}\right)_{i}=v_{i}^{\prime} \text { for } i \in\{m, m+1\}, \\
& \left(\chi_{\{m, m+1\}} v^{\prime}\right)_{i}=0 \text { for } i \notin\{m, m+1\} .
\end{aligned}
$$

Proceeding from (4.29), (5.4), (5.6) we obtain that, under our assumptions, system $(5.11)$ is uniquely solvable for $v_{m}^{\prime}, \ldots, v_{m+1}^{\prime}$ in $L^{2}(\mathbb{C})$ (by the method of successive approximations) and that, in addition, $v_{m}^{\prime}, \ldots, v_{m+1}^{\prime}$ belong to $L^{p}(\mathbb{C})$ if $p$ is sufficiently close to 2 .

In addition, using $(5.12)$ one can see that $v^{\prime} \in L^{p}(\mathbb{C})$ if $p$ is sufficiently close to 2 .

Using that

$$
\begin{aligned}
& v \in C(\mathbb{C}), \quad|v(z)| \rightarrow 0 \text { as }|z| \rightarrow \infty, \\
& \text { for } v=\left(\bar{T} v_{1}^{\prime}, \ldots, \bar{T} v_{m}^{\prime}, \quad T v_{m+1}^{\prime}, \ldots, T v_{2 m}^{\prime}\right),
\end{aligned}
$$

where $T, \bar{T}$ are the operators of $(3.27),(3.28)$ and $v^{\prime} \in L^{p}(\mathbb{C})$ if $p$ is sufficiently close to 2 , one can complete the proof of Lemma 3.1.

\section{Proof of Theorem 3.2}

Proceeding from (4.22), (3.14), (3.18) we obtain that

$$
\begin{aligned}
& v_{j}=\bar{T} \sum_{k=1}^{j}(-\bar{\Pi})^{j-k} w_{2(m-k+1)} f, \quad j=1, \ldots, m, \\
& v_{j}=T \sum_{k=1}^{2 m-j+1}(-\Pi)^{2 m-j+1-k} w_{-2(m-k+1)} f, \quad j=m+1, \ldots, 2 m,
\end{aligned}
$$

where $T, \Pi, \bar{T}, \bar{\Pi}$ are the operators of (3.27), (3.28). In addition, due to (4.22), (3.14) we have also that

$$
\partial_{z} v_{m+1}(z)+\partial_{\bar{z}} v_{m}(z)+F_{0}(z)=w_{0}(z) f(z), \quad z \in \mathbb{C} .
$$

In turn, formulas (6.1a) for $j=m,(6.1 \mathrm{~b})$ for $j=m+1$ and formula (6.2) imply that

$$
w_{0} f+\sum_{l=1}^{m}\left((-\bar{\Pi})^{l} w_{2 l}+(-\Pi)^{l} w_{-2 l}\right) f=F_{0} .
$$

Using (5.4a), (6.3) one can see, in particular, that under our assumptions,

$$
F_{0} \in L^{p}(\mathbb{C}) \text { for each } p>1 \text {. }
$$

Equation (3.29) follows from formula (6.3), the definitions of $F$ and $\tilde{w}_{2 l}$ and formula (4.26). In addition, using (4.29) we obtain that

$$
\begin{aligned}
& \left\|\sum_{l=1}^{m}\left((-\bar{\Pi})^{l} \tilde{w}_{2 l}+(-\Pi)^{l} \tilde{w}_{-2 l}\right) \chi_{D}\right\|_{L^{2}(\mathbb{C}) \rightarrow L^{2}(\mathbb{C})} \leq \\
& \sum_{l=1}^{m}\left(\sup _{z \in D}\left|\tilde{w}_{2 l}(z)\right|+\sup _{z \in D}\left|\tilde{w}_{-2 l}(z)\right|\right) .
\end{aligned}
$$


Inequality (6.5) completes the proof of Theorem 3.2.

\section{References}

[ Ba1] G. Bal, On the attenuated Radon transform with full and partial measurements, Inverse Problems 20 (2004), 399-418

[ Ba2] G. Bal, Inverse transport theory and applications, Inverse Problems 25 (2009), 053001 (48pp)

[ Bo1] J. Boman, An example of non-uniqueness for a generalized Radon transform, J. Anal. Math. 61 (1993), 395-401

[ Bo2] J. Boman, Local non-injectivity for weighted Radon transforms, Contemporary Mathematics 559 (2011), 39-47

[BQ] J. Boman and E.T. Quinto, Support theorems for real-analytic Radon transforms, Duke Math. J. 55 (1987), 943-948

[ BS] J. Boman and J.O. Strömberg, Novikov's inversion formula for the attenuated Radon transform - a new approach, J.Geom.Anal. 14 (2004), 185-198

[ Ch] L.T. Chang, A method for attenuation correction in radionuclide computed tomography, IEEE Trans. Nucl. Sci. NS-25 (1978), 638-643

[Fi] D.V. Finch, Uniqueness for the attenuated X-ray transform in the physical range, Inverse Problems 2 (1986), 197-203

[ FN] A.S. Fokas and R.G. Novikov, Discrete analogues of $\bar{\partial}-$ equation and of Radon transform, C. R. Acad. Sci. Paris Sér. I Math. 313 (1991), 75-80

[ G] S. Gindikin, A remark on the weighted Radon transform on the plane, Inverse Problems and Imaging 4 (2010), 649-653

[ GN] J.-P. Guillement and R.G. Novikov, Optimized analytic reconstruction for SPECT, J. Inv. Ill-Posed Problems, doi: 10.1515/jip-2012-0011

[ Ku] L.A. Kunyansky, Generalized and attenuated Radon transforms: restorative approach to the numerical inversion, Inverse Problems 8 (1992), 809-819

[ MP] E.X. Miqueles and A.R. De Pierro, Fluorescence tomography: reconstruction by iterative methods, ISBI (2008), 760-763

[ Na] F. Natterer, The Mathematics of Computerized Tomography (Stuttgart: Teubner), 1986

[No1] R.G. Novikov, An inversion formula for the attenuated X-ray transformation, Ark. Mat. 40 (2002), 145-167

[No2] R.G. Novikov, Weighted Radon transforms for which Chang's approximate inversion formula is exact, Uspekhi Mat. Nauk 66 (2) (2011), 237-238

[ Q] E.T. Quinto, The invertibility of rotation invariant Radon transforms, J. Math. Anal. Appl. 91 (1983), 510-522

[ Ra] J. Radon, Uber die Bestimmung von Funktionen durch ihre Integralwerte langs gewisser Mannigfaltigkeiten, Ber. Verh. Sachs. Akad. Wiss. Leipzig, Math-Nat., K 169 (1917), 262-267

[ Ri] M. Riesz, Sur le maxima des formes billinéares et sur les fonctionnelles linéaires, Acta Math. 49 (1928), 465-497

[ TM] O.J. Tretiak and C. Metz, The exponential Radon transform, SIAM J. Appl. Math. 39 (1980), 341-354

[ V] I.N. Vekua, Generalized Analytic Functions, Pergamon Press Ltd. 1962 